\title{
Strong Resonance Effect in a Lossy Medium-Based Optical Cavity for Angle Robust Spectrum Filters
}

\author{
Kyu-Tae Lee, Sungyong Seo, Jae Yong Lee, and L. Jay Guo*
}

Color filters have been widely used as key elements in applications ranging from light emitting diodes/image sensors to liquid crystal display technology. The color filters, based on plasmonic nanostructures and photonic subwavelength gratings, have emerged as attractive alternatives to address disadvantages of conventional color filters utilizing organic dyes. ${ }^{[1-5]}$ Conventional organic dye based filters are susceptible to environmental factors such as longstanding ultraviolet illumination and high temperature, which will cause performance degradation over time. ${ }^{[6]}$ However, the biggest challenge facing both the plasmonic and photonic nanostructure based color filters is that the light at oblique angle of incidence leads to the shift in a resonance, thus creating different colors. ${ }^{[1-5,7-10]}$ This is because these works rely on the grating coupling in order to excite the plasmonic or photonic mode, showing innately angle dependent property that is due to the momentum matching conditions. Such angle dependent spectral characteristic is a significant drawback, which makes the filters difficult to be applicable in practical usage. To address this issue, angle-insensitive structural colors employing metallic nanocavity have been proposed, which involves directing the incident optical energy toward deep subwavelength grooves. ${ }^{[11]}$ However, it requires a number of fabrication steps and nanopatterning, which can be difficult to scale to large area for many practical applications.

Strong interference effects in a lossy medium have recently been demonstrated, allowing the thickness of the cavity layer to be remarkably reduced as compared to that of traditional cavity consisting of a transparent material. ${ }^{[12,13]}$ This is enabled by the large absorption coefficient of the lossy material and the non-trivial phase change from the reflection at the boundary between the lossy material and metal. With these properties, a resonance behavior was observed in the structure that is composed of a few nanometer thick germanium (Ge) layer on top of the gold $(\mathrm{Au})$ substrate. However, owing to the presence of the interband transition of $\mathrm{Au}(470 \mathrm{~nm})$ and the band gap of $\mathrm{Ge}$ $(0.66 \mathrm{eV})$, both of which can lead to the strong absorption at visible frequencies, the bandwidth and the efficiency of the device are broad and low, showing poor quality factor (Q-factor) and hence low color purity. ${ }^{[14,15]}$ Additionally, the reflection at the interface between air and Ge is weak that can make the bandwidth even broader.

\author{
K.-T. Lee, S. Seo, J. Y. Lee, Prof. L. J. Guo \\ Department of Electrical \\ Engineering and Computer Science \\ University of Michigan \\ Ann Arbor, Michigan 48109, USA \\ E-mail: guo@umich.edu
}

DOI: 10.1002/adma.201402117
Herein, we present high color purity reflective spectrum filters based on a nanoresonator comprising ultra-thin highly absorbing layer sandwiched by two metals. The significantly improved color purity is achieved by two strategies to enhance the Q-factor of the nanoresonator: 1) choosing a highly absorbing material that has a lower complex refractive index than $\mathrm{Ge}$, and 2) putting additional optically thin metal layer on top of the structure to increase the reflection. In addition, such structures are capable of producing specific reflection colors (e.g., cyan, magenta, and yellow) that are insensitive to the angle of incidence up to $\pm 65^{\circ}$. Lastly, the influence of the metallic substrates on the resonance behavior was also investigated.

The structure of proposed spectrum filters is schematically shown in Figure 1(a), consisting of a highly absorbing material sandwiched by a top thin and bottom thick metal layer built on a fused silica substrate. Amorphous silicon (a-Si) is chosen as the lossy material since the complex refractive index of a-Si is lower than that of Ge. This relatively low index leads to the low light absorption $\left(\frac{2 \pi c \varepsilon_{0} n k}{\lambda}|E(x, \lambda)|^{2}\right.$, where $c$ : speed of light, $\varepsilon_{0}$ : permittivity of free space, $n$ : real part of the refractive index, $k=\frac{\lambda \alpha}{4 \pi}$ : extinction coefficient, $\alpha$ : absorption coefficient), thereby increasing the Q-factor, while still maintaining the strong interference effects in the ultra-thin regimes. ${ }^{[16,17]}$ To investigate the chromaticity of the proposed filters, we performed a simulation of the structure that is decomposed of highly absorbing material on different metallic substrates as illustrated in Figure 1(b). As alluded to previously, Ge (9 nm) on $\mathrm{Au}$ substrate (\#1, black solid line) shows the most broad bandwidth mainly due to the interband transition of $\mathrm{Au}$ and the large absorption of $\mathrm{Ge}$ in the visible regimes. In comparison, a-Si whose absorption is relatively weak on silver (Ag) substrate enables the bandwidth to be sharpened (\#2, red solid line). Since the absorption of $\mathrm{Au}$ is not significant at $550 \mathrm{~nm}$ that is far from the interband transition of $\mathrm{Au}$, the purity of the magenta color of \#3 (blue solid line) is similar with that of \#2. By putting additional top thin metal layer (\#4, dark yellow solid line), the color coordinate moves toward the outward direction showing significantly improved color purity as compared with the case of \#1 (Ge on Au substrate) as can be seen in Figure 1(c). Similar studies for the yellow and cyan colors can be found in the Supporting Information. The calculated full width at half maximum (FWHM) and the corresponding Q-factor of each optical cavity for the CMY colors are summarized in Table I given in the Supporting Information. We note that the thickness of the a-Si layer is different for \#2 (16 nm) and \#4 (20 nm) in Figure 1(b). This is because the phase shift occurring upon the reflection from the interface between air and a-Si is larger than that of $\mathrm{Ag} / \mathrm{a}-\mathrm{Si}$ interface as described in the Figure S2. 
(a)

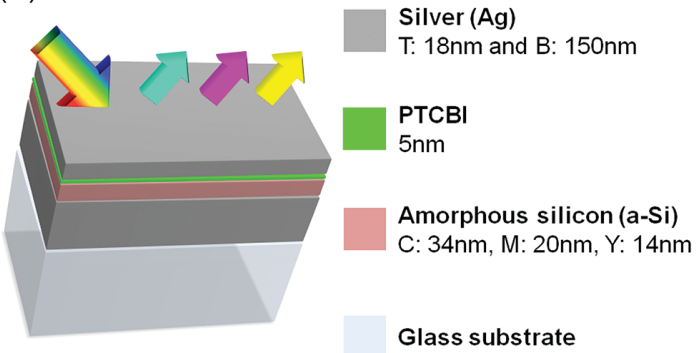

(b)

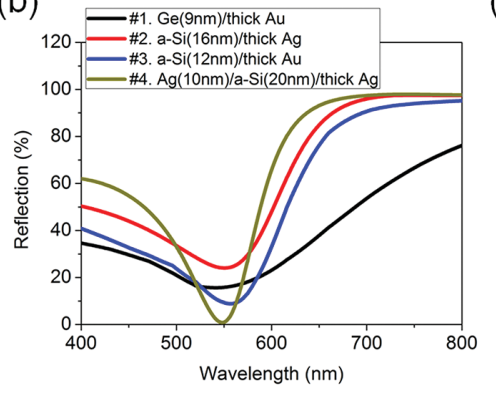

(c)

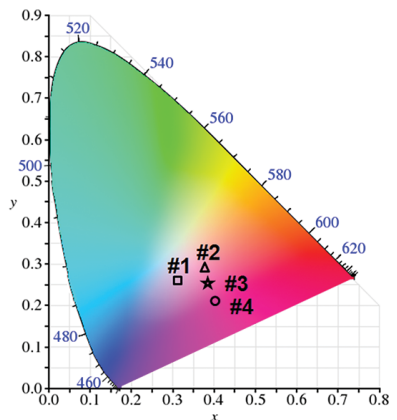

(d)

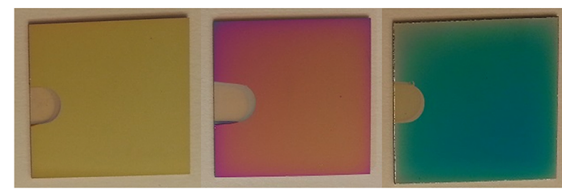

(e)

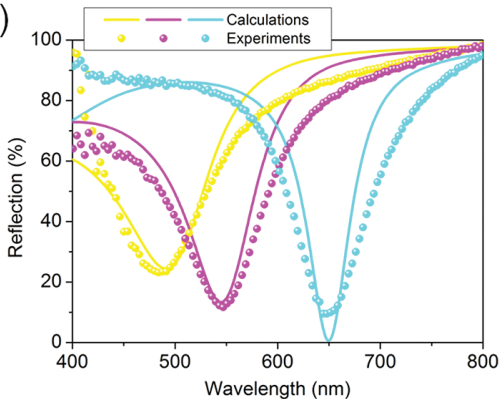

(f)

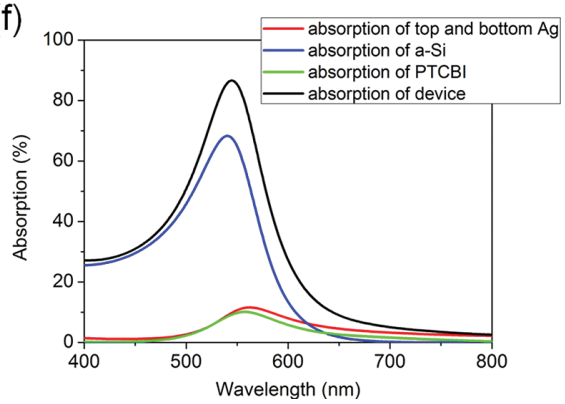

Figure 1. (a) A schematic view of the proposed angle robust spectrum filters. (b) Calculated reflection spectra of several cavities that consist of different semiconductor and metallic substrate. (c) Calculated color coordinates of (b) exhibiting how the color purity is improved. (d) A photograph of the fabricated devices at normal incidence. (e) Calculated and measured reflection spectra at normal incidence. (f) Comparison of the absorption spectrum of whole device to the absorption profile of other rest layers.

Thus, the thicker a-Si film thickness is required with the top Ag layer in order to get the same resonance position, i.e. when the net shift of the propagation phase and two reflection phases is equal to $2 \mathrm{~m} \pi$.

In device fabrication, the Ag is employed for both top and bottom metal layers due to its lowest material absorption loss and highest reflectivity across the whole visible wavelength ranges among all metals. The thickness of the top $\mathrm{Ag}$ film is designed to be $18 \mathrm{~nm}$ that allows the incident light to pass through the structure and simultaneously provides an enhanced reflection from within the resonance cavity, whereas the bottom $\mathrm{Ag}$ is $150 \mathrm{~nm}$ to block the transmitted light. It should be noted that as the thickness of the top metal layer increases, the spectral bandwidth of the resonance reduces leading to improved color purity, however with decreased efficiency in the reflected colors. An organic layer, perylenetetracarboxylic bis-benzimidazole (PTCBI), is used to ensure a smooth thin Ag film that minimizes the scattering. ${ }^{[18]} \mathrm{Ag}$ and PTCBI were deposited by thermal evaporation and a-Si was deposited by plasma-enhanced chemical vapor deposition (PECVD). The complex refractive indices of $\mathrm{Ag}(20 \mathrm{~nm}), \mathrm{a}-\mathrm{Si}(50 \mathrm{~nm}), \mathrm{Si}_{3} \mathrm{~N}_{4}$ $(100 \mathrm{~nm})$, and PTCBI $(10 \mathrm{~nm})$ as a function of wavelength were measured by a spectroscopic ellipsometer (M-2000, J. A. Woollam) and illustrated in Figure S3. The thicknesses of the a-Si layer for producing cyan, magenta, and yellow (CMY) colors are found to be 34,20 , and $14 \mathrm{~nm}$ and the corresponding resonances (i.e., reflection dip or absorption peak) are 650,545 , and $490 \mathrm{~nm}$, respectively. The simulated reflection spectra (solid lines) at normal incidence calculated by a transfer matrix method are depicted in Figure 1(e) that matches well with the experimental results (solid symbols) measured using a spectrometer (HR4000CG, Ocean Optics). There is a slight difference between the measured and calculated results, which is attributed to the non-even surface of each layer. This results in a scattering loss and hence produces broader profiles. It is obvious that the reflection spectrum of the cyan color shows the narrowest bandwidth among the three colors, exhibiting $74 \mathrm{~nm}$ (the corresponding FWHM from the simulation: $58 \mathrm{~nm}$ ) of FWHM. As there is no transmitted light due to an optically thick metallic substrate, the complementary spectrum of the reflection profile is the absorption spectrum, both of which exhibit the narrowest FWHM from the cyan device. This is because the imaginary part of the refractive index of the a-Si reduces as the wavelength increases, yielding increased Q-factor. On the contrary, the yellow device shows the broadest FWHM that is attributed to the large absorption of a-Si material at shorter wavelengths. Note that the FWHMs of the yellow and magenta colored devices obtained from the experiment (simulation) are $136 \mathrm{~nm}(122 \mathrm{~nm})$ and $114 \mathrm{~nm}(98 \mathrm{~nm})$, respectively. Figure 1(d) shows a photograph of the fabricated CMY devices at normal incidence. In Figure 1(f), the simulated absorption profile of whole device is plotted together with the absorption spectrum of each layer for comparison. As can be seen from the figure, the absorption is primarily determined by the a-Si layer due to the large refractive index as compared to that of other layers (See Figure S3). Moreover, it is apparent that an absorption peak is observed for all the layers near the resonance because the incident light can traverse each layer more times than the off-resonance condition, hence giving rise to higher absorption.

To explore the incident angle dependence of such spectrum filters, angle resolved reflection spectra for transverse magnetic (TM) and transverse electric (TE) polarization are simulated by the transfer matrix method (solid lines) and the corresponding 
(a)

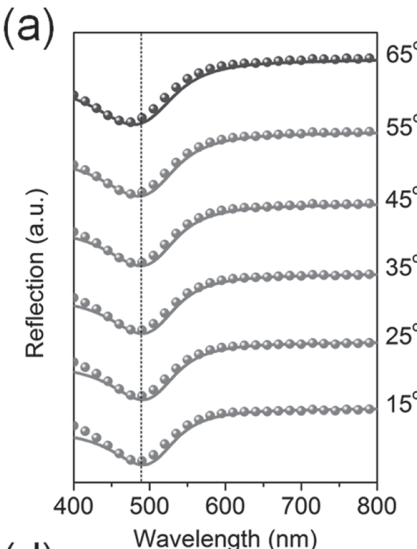

(d)

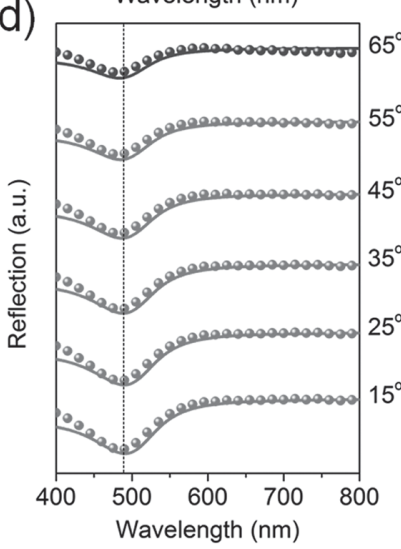

(b)

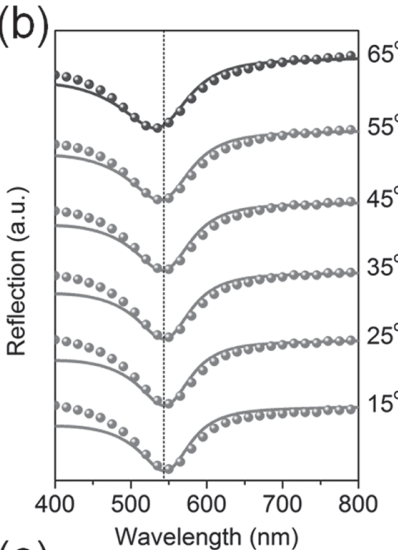

(e)

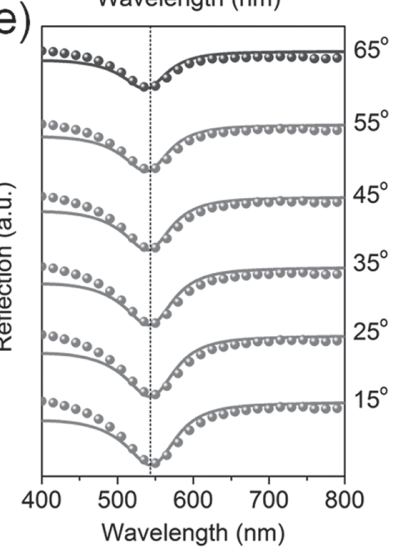

www.MaterialsViews.com

(c)

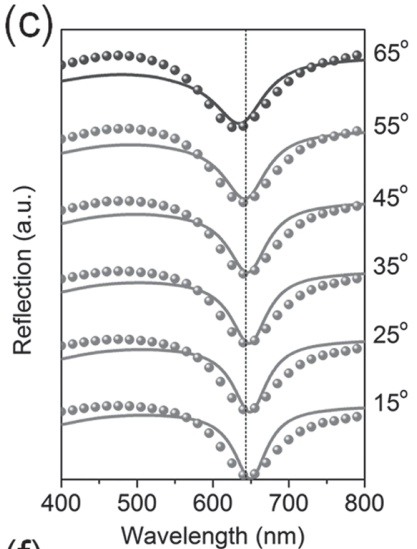

(f)

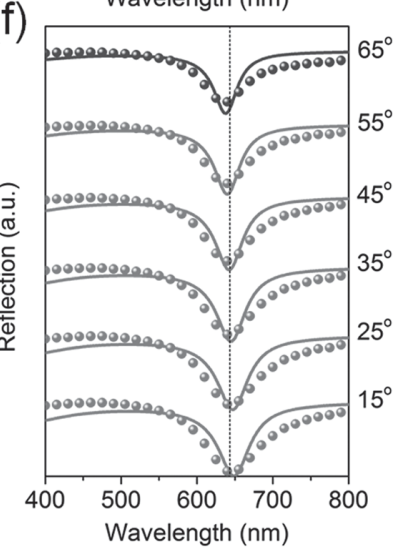

(g)
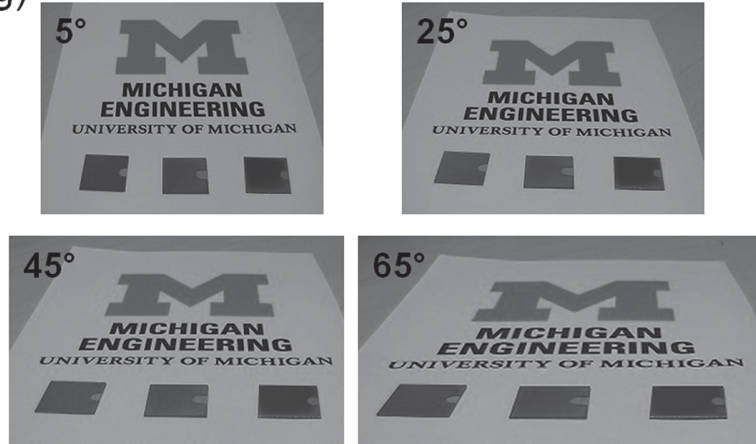

Figure 2. Measured and calculated reflection spectra at oblique angles of incidence ranging from $15^{\circ}$ to $65^{\circ}$ for TM (a-c) and TE polarization (d-f). (a,d) A resonance occurs at $485 \mathrm{~nm}$ corresponding to yellow. (b,e) $545 \mathrm{~nm}$ corresponding to magenta. (c,f) $645 \mathrm{~nm}$ corresponding to cyan. (g) Optical images of the fabricated spectrum filters from $5^{\circ}$ to $65^{\circ}$. It is clear that there is no color change even at large angle $\left(65^{\circ}\right)$, validating the angle robust property of our proposed device. (For color version see $\mathrm{SI}$ )

measured profiles (solid symbols) are obtained by a variable angle spectroscopic ellipsometer (V-VASE, J. A. Woollam) for incident angles of $15^{\circ}$ to $65^{\circ}$ as exhibited in Figure $2(\mathrm{a})-(\mathrm{f})$. Calculation results show good agreement with the experiment data. The resonance wavelength remains invariance over a wide range of incident angles for all three colors for both polarizations. We note that the position of the resonance is still maintained while the intensity gets reduced at larger angles than $65^{\circ}$. The calculated and measured angle resolved reflection spectra for unpolarized light are also given in the Supporting Information. Optical images in Figure 2(g) were taken with an indoor ambient light at four different angles showing no color change. The size of the fabricated spectrum filters is $2 \mathrm{~cm} \times$ $2 \mathrm{~cm}$. The capability of such angle insensitivity could be potentially useful in light emitting devices, image sensors, and display applications.

Such angle-invariant resonance behavior is in stark contrast to that in a normal Fabry-Perot (F-P) resonator, e.g. by sandwiching a transparent medium between two reflecting metal films. Previous studies of structures containing ultrathin absorbing material on top of a metal have attributed the angle-insensitive behavior to the negligible propagation phase in the ultra-thin dielectric layer. ${ }^{[12,13]}$ However as is shown by the detailed calculation, even though the propagation phase 

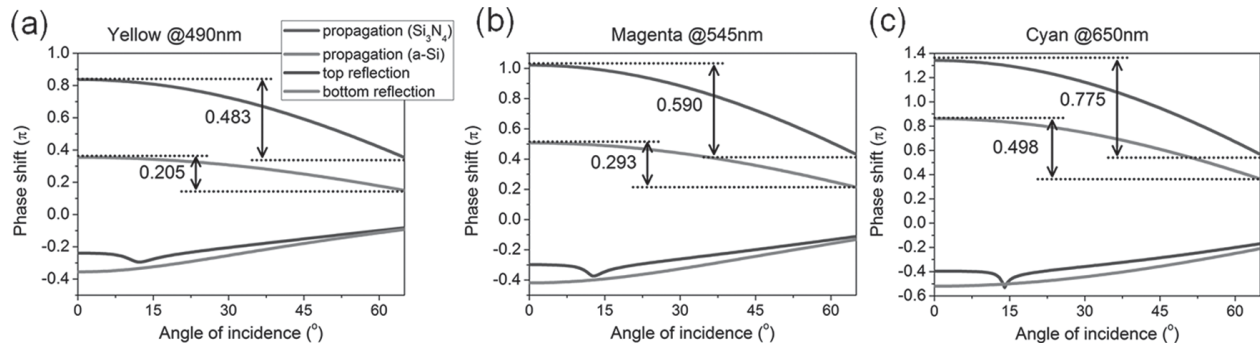

Figure 3. (a-c) Calculated non-trivial reflection phase shifts at both the top and bottom semiconductor and metal interfaces and the accumulated phase shift during the propagation as a function of the angle of incidence for (a) Yellow corresponding to $490 \mathrm{~nm}$. (b) Magenta corresponding to $545 \mathrm{~nm}$. (c) Cyan corresponding to $650 \mathrm{~nm}$. The propagation phase shift attained from an optically transparent cavity $\left(\mathrm{Si}_{3} \mathrm{~N}_{4}\right)$ is included as a reference. (For color version see SI)

in an ultra-thin absorbing material is small than of a normal transparent medium, it is non-trivial and show the same angledependent behavior. Therefore the ultra-thin absorbing material alone cannot explain the peculiar angle-independence effect. Figure 3(a)-(c) display the angle dependent propagation phase and the phase shift when light reflects from the two metal surfaces. These results are obtained by using the transfer matrix method. As seen in the figures, the phase due to the light propagation in the ultra-thin highly absorbing material is about half of that in an optically transparent material (e.g., $\mathrm{Si}_{3} \mathrm{~N}_{4},[n=2$ and $k=0$ at visible frequency]), and decreases with increasing angle. Note that the same metal thicknesses as in the fabricated structures are used for the reference structure $\left(\mathrm{Ag} / \mathrm{Si}_{3} \mathrm{~N}_{4} / \mathrm{Ag}\right)$ in order to establish the same resonance wavelength, and the thickness of the $\mathrm{Si}_{3} \mathrm{~N}_{4}$ layer is chosen as 109,83 , and $68 \mathrm{~nm}$ respectively to create the CMY colors. The $\mathrm{Si}_{3} \mathrm{~N}_{4}$ layer is thicker than that of a-Si so as to obtain the same resonance position. As we explained previously, the strong resonance in the ultra-thin thickness of a-Si layer is enabled by the non-trivial reflection phase shifts. In the case of reference structure where a transparent medium sandwiched by two metal films, the phase shift of light reflecting from the metal surface is close to and with weak angle-dependence. Therefore the F-P cavity containing transparent medium will show the typical angle-dependent resonance shift. In the case of a highly absorbing medium, the phase shift of light reflecting from the metal surface is very different from but non-trivial, and varies with the angle of incidence. But interestingly, the phase change due to propagation in the lossy medium and those due to the reflections from the metal mostly cancels out at all angles, leading to a close-to-zero phase in the resonator!

From physics point of view, the phase shift of light reflecting from a metal is related to the light penetration into the metal at the visible band. In Figure 4(a), the calculated reflection spectra from three metallic substrates $(\mathrm{Ag}, \mathrm{Al}$, and $\mathrm{Au})$ are displayed. To create a resonance at the same wavelength of $550 \mathrm{~nm}$, it is found that different thickness of a-Si layer is required. This is due to the different skin depth $(\delta)$ of these metals. For example, $\delta$ for $\mathrm{Al}$ and $\mathrm{Au}$ are about $13 \mathrm{~nm}$ and $31 \mathrm{~nm}$ at $620 \mathrm{~nm}$, respectively. ${ }^{[19]} \delta$ of $\mathrm{Ag}$ has an intermediate value of $\sim 22 \mathrm{~nm}$, between that of $\mathrm{Al}$ and $\mathrm{Au} .{ }^{[20]}$ Since light can penetrate deeper into $\mathrm{Au}$ at this wavelength than those of $\mathrm{Al}$ and $\mathrm{Ag}$, the thickness of a-Si layer needed to establish the same resonance condition is correspondingly smaller $(17 \mathrm{~nm})$ that that in the case of Al substrate $(26 \mathrm{~nm})$. This phenomenon can also be seen by examining the normalized electric field distributions at the resonance $(550 \mathrm{~nm})$ for the different metallic substrates as shown in Figure 4(b): the amplitude of the electric field entering into the $\mathrm{Au}$ is much larger than the $\mathrm{Ag}$ and $\mathrm{Al}$, which is due to the longer penetration depth of the Au.

In summary, we have presented angle robust reflective spectrum filters with high color purity exploiting strong resonance effects in a F-P optical cavity containing lossy media. Significantly enhanced color purity is attained by choosing the absorbing material that possesses the lower complex dielectric constant and by inserting additional top thin Ag layer. The reflection spectrum with narrow bandwidth $(\sim 74 \mathrm{~nm})$ is achieved from the fabricated spectrum filter, which shows good agreement with the simulated profiles. Besides, the devices demonstrate the angle robust characteristic up to $\pm 65^{\circ}$ that can address a crucial challenge in current plasmonic and photonic based spectrum filters, potentially opening the door to various applications such as light emitting devices, display technologies, and image sensors.
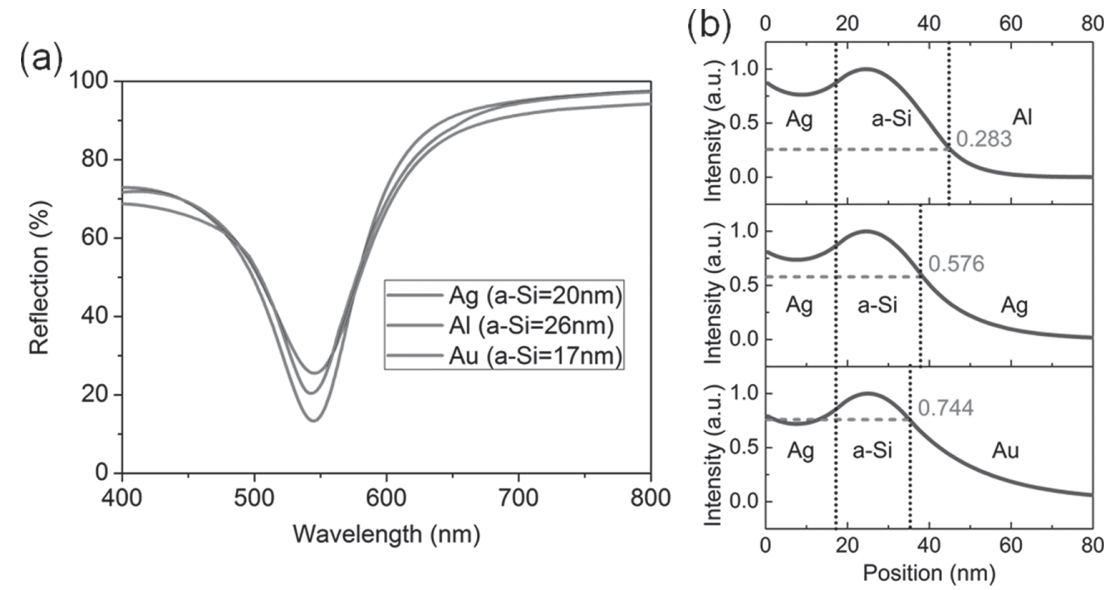

Figure 4. (a) Calculated reflection spectra obtained from different metallic substrates. (b) Normalized intensity distributions of the electric field at $550 \mathrm{~nm}$ for different metallic substrates. 


\section{Experimental Section}

Device Fabrication: Before starting a deposition, a fused silica substrate was cleaned by using the piranha solution (mixture of $\mathrm{H}_{2} \mathrm{SO}_{4}$ and $\mathrm{H}_{2} \mathrm{O}_{2}$ with 1:1 volume ratio) for $30 \mathrm{~min}$. The $150 \mathrm{~nm}$ of $\mathrm{Ag}$ was deposited by a thermal evaporation at a rate of $1 \mathrm{As}^{-1}$ with a high vacuum $\left(1 \times 10^{-6} \mathrm{mbar}\right)$. Then, a PECVD tool was used for the deposition of an ultra-thin a-Si layer at a rate of $0.6 \mathrm{As}^{-1}\left(\mathrm{SiH}_{4}\right.$ gas with $13.56 \mathrm{MHz}$ RF power). Finally, the device structure was completed by thermally evaporating a wetting layer, PTCBI $(5 \mathrm{~nm})$, and then the $18 \mathrm{~nm}$ of Ag.

Simulation and Measurement: A transfer matrix method was used for the optical simulation and all the refractive indices used in the simulation were measured by a spectroscopic ellipsometer (M-2000, I. A. Woollam). The reflection spectrum of the fabricated devices at normal incidence was measured by using a spectrometer (HR4000CG, Ocean Optics) for TM polarization. And the angle resolved reflection spectrum measurement was performed by using a variable angle spectroscopic ellipsometer (VASE, J. A. Woollam).

\section{Supporting Information}

Supporting Information is available from the Wiley Online Library or from the author.

\section{Acknowledgements}

The authors would like to acknowledge the support by the National Science Foundation Grant No. ECCS 1202046. The authors would also like to thank Kyusang Lee for angle measurements.

Received: May 11, 2014

Revised: June 28, 2014

Published online: July 28, 2014
[1] Y. Kanamori, M. Shimono, K. Hane, IEEE Photon. Technol. Lett. 2006, 18, 2126

[2] Y.-T. Yoon, H.-S. Lee, S.-S. Lee, S. H. Kim, J.-D. Park, K.-D. Lee, Opt. Express 2008, 16, 2374.

[3] T. Xu, Y.-K. Wu, X. Luo, L. J. Guo, Nat. Commun. 2010, 1, 59.

[4] K. Diest, J. A. Dionne, M. Spain, H. A. Atwater, Nano Lett. 2009, 9, 2579.

[5] C.-H. Park, Y.-T. Yoon, S.-S. Lee, Opt. Express 2012, 20, 23769

[6] R. W. Sabnis, Displays 1999, 20, 119.

[7] T. Xu, H. Shi, Y.-K. Wu, A. F. Kaplan, J. G. Ok, L. J. Guo, Small 2011, 7, 3128.

[8] Y. S. Do, J. H. Park, B. Y. Hwang, S.-M. Lee, B.-K. Ju, K. C. Choi, Adv. Opt. Mater. 2013, 1, 133.

[9] H. J. Park, T. Xu, J. Y. Lee, A. Ledbetter, L. J. Guo, ACS Nano 2011, 5, 7055.

[10] A. F. Kaplan, T. Xu, L. J. Guo, Appl. Phys. Lett. 2011, 99, 143111.

[11] Y.-K. R. Wu, A. E. Hollowell, C. Zhang, L. J. Guo, Sci. Rep. 2013, 3, 1194; DOI: 10.1038/srep01194.

[12] M. A. Kats, R. Blanchard, P. Genevet, F. Capasso, Nature Mater. 2013, 12, 20

[13] M. A. Kats, S. J. Byrnes, R. Blanchard, M. Kolle, P. Genevet, J. Aizenberg, F. Capasso, Appl. Phys. Lett. 2012, 103, 101104.

[14] P. G. Etchegoin, E. C. Le Ru, M. Meyer, J. Chem. Phys. 2006, 125, 164705.

[15] M. El Kurdi, S. David, X. Checoury, G. Fishman, P. Boucaud, O. Kermarrec, D. Bensahel, B. Ghyselen, Opt. Commun. 2008, 281, 846.

[16] L. A. A. Pettersson, L. S. Roman, O. Inganas, J. Appl. Phys. 1999, 86, 487.

[17] J. Y. Lee, K.-T. Lee, S. Seo, L. J. Guo, Sci. Rep. 2014, 4, 4192.

[18] K.-T. Lee, J. Y. Lee, S. Seo, L. J. Guo, unpublished.

[19] P. Biagioni, J.-S. Huang, B. Hecht, Rep. Prog. Phys. 2012, 75, 024402.

[20] S. O'Brien, D. McPeake, S. A. Ramakrishna, J. B. Pendry, Phys. Rev. B 2004, 69, 241101 . 